\title{
Neuroprotective effects of caffeic acid phenethyl ester against sevoflurane-induced neuronal degeneration in the hippocampus of neonatal rats involve MAPK and PI3K/Akt signaling pathways
}

\author{
LI-YAN WANG ${ }^{1}$, ZHI-JUN TANG ${ }^{2}$ and YU-ZENG HAN ${ }^{3}$ \\ Departments of ${ }^{1}$ Pediatric Surgery, ${ }^{2}$ Orthopedics in Repair and Reconstruction and ${ }^{3}$ Pediatric Internal Medicine, \\ Linyi People's Hospital, Linyi, Shandong 276003, P.R. China
}

Received May 30, 2015; Accepted June 24, 2016

DOI: $10.3892 / \mathrm{mmr} .2016 .5586$

\begin{abstract}
Millions of infants and children are exposed to anesthesia every year during medical care. Sevoflurane is a volatile anesthetic that is frequently used for pediatric anesthesia. However, previous reports have suggested that the administration of sevoflurane promotes neurodegeneration, raising concerns regarding the safety of its usage. The present study aimed to investigate caffeic acid phenethyl ester (CAPE) and its protective effect against sevoflurane-induced neurotoxicity in neonatal rats. Rat pups were administered with CAPE at 10,20 or $40 \mathrm{mg} / \mathrm{kg}$ body weight from postnatal day $1(\mathrm{P} 1)$ to P15. The P7 rats were exposed to sevoflurane (2.9\%) for $6 \mathrm{~h}$. Control group rats received no sevoflurane or CAPE. Neuronal apoptosis was determined by terminal deoxynucleotidyl transferase dUTP nick-end labeling assay. The expression levels of caspases (caspase-3, -8 and -9), apoptotic pathway proteins [Bcl-2-associated X protein (Bax), B cell CCL/lymphoma 2 (Bcl-2), Bcl-2-like 1 (Bcl-xL), Bcl-2-associated agonist of cell death (Bad) and phosphorylated (p)-Bad], mitogen-activated protein kinases (MAPK) signaling pathway proteins [c-Jun N-terminal kinase (JNK), p-JNK, extracellular signal-regulated kinase (ERK)1/2, p-ERK1/2, p38, p-p38 and p-c-Jun] and the phosphoinositide 3-kinase (PI3K)/Akt cascade were evaluated by western blotting following sevoflurane and CAPE treatment. In addition, the expression of cleaved caspase-3 was analyzed by immunohistochemistry. CAPE significantly reduced sevoflurane-induced apoptosis, downregulated the expression levels of caspases and pro-apoptotic proteins (Bax and $\mathrm{Bad}$ ) and elevated the expression levels of $\mathrm{Bcl}-2$ and $\mathrm{Bcl}-\mathrm{xL}$ when compared with sevoflurane treatment.
\end{abstract}

Correspondence to: Dr Yu-Zeng Han, Department of Pediatric Internal Medicine, Linyi People's Hospital, 27 Jiefang Road, Linyi, Shandong 276003, P.R. China

E-mail: hanyuzheng@hotmail.com

Key words: apoptosis, caffeic acid phenethyl ester, mitogen-activated protein kinases, phosphoinositide 3-kinase/Akt, neuroprotective, sevoflurane
Furthermore, CAPE appeared to modify the expression levels of MAPKs and activate the PI3K/Akt signaling pathway. Thus, the present study demonstrated that CAPE effectively inhibited sevoflurane-induced neuroapoptosis by modulating the expression and phosphorylation of apoptotic pathway proteins and MAPKs, and by regulating the PI3K/Akt pathway.

\section{Introduction}

Volatile anesthetics are frequently used during pediatric surgery (1). Sevoflurane [2,2,2-trifluoro-1-(trifluorome thyl) ethyl fluoro methyl ether] is widely administered as a general anesthetic in pediatric patients, due to its fast induction and recovery times, and it causes less irritation to the airways compared with other inhaled anesthetics (2). Accumulating evidence indicates that volatile anesthetics induce neuronal apoptosis (3-6) and affect neurogenesis in vitro and in vivo $(7,8)$. Furthermore, long-term neurocognitive function was observed to be altered in 7 day-old rats (9).

Children aged $<4$ years that were exposed to general anesthesia more than once have an increased risk of developing learning disabilities $(10,11)$. Although sevoflurane is not as cytotoxic as isoflurane and desflurane, sevoflurane exposure increases the risk of neurodevelopmental impairments and cognitive dysfunction in neonatal animal models (12-14).

The various mechanisms that underlie anesthetic-mediated neuronal apoptosis in the evolving brain are yet to be fully determined and numerous potential mechanisms have been proposed, including: i) Disruption of intracellular calcium homeostasis (15-17); ii) regulation of the cell cycle (18); iii) inhibition of N-methyl-D-aspartate receptors and activation of gamma-aminobutyric acid receptors; and iv) associated impairment of synaptogenesis (19-22).

Mitogen-activated protein kinases (MAPKs) are a group of serine-threonine protein kinases that are important during neurogenesis (23), neurodegeneration (24) and brain inflammation (25). The major members of the MAPK family are c-Jun N-terminal kinase (JNK), extracellular signal-regulated kinase (ERK) and p38 MAPK. Previous studies have demonstrated an association between MAPK signaling pathways and neurotoxicity induced by anesthetics. Wang et al (26) reported that $\mathrm{N}$-stearoyl-1-tyrosine protects the developing 
brain against sevoflurane-induced neurotoxicity by regulating the ERK1/2 signaling pathway. Furthermore, dexmedetomidine was demonstrated to regulate the phosphorylation levels of ERK1/2 in the neonatal rat brain (27) and provided neuroprotection against isoflurane-induced neurodegeneration in the hippocampus of neonatal rats by modulating the phosphoinositide 3-kinase (PI3K)/Akt signaling pathway (28). Zhao et al (29) reported that isoflurane causes neurodegeneration via apoptosis through excessive activation of inositol 1,4,5-trisphosphate receptors ( $\mathrm{InsP}_{3} \mathrm{Rs}$ ).

Strategies to potentially reduce the anesthetic-induced neurotoxicity require further research. Previous investigations focused on using plant-derived compounds for the therapy of various medical conditions. Resveratrol, a phenolic antioxidant present in grapes and berries, was demonstrated to protect neuronal cells from isoflurane-induced cytotoxicity by regulating the Akt signaling cascade (30). Caffeic acid phenethyl ester (CAPE) is a phenolic chemical compound present in numerous plants and is extracted from honeybee hive propolis (31). It is a strong antioxidant (32), and also exhibits anti-proliferative (33) and anti-inflammatory effects (32,34). Furthermore, the neuroprotective effects of CAPE in in vivo and in vitro experimental models have been demonstrated (35-37).

Thus, considering the biological effects of CAPE, the present study aimed to investigate whether CAPE protects against sevoflurane-induced neurotoxicity in a neonatal rat model.

\section{Materials and methods}

Study animals. The present study was approved by the animal care and ethical committee of Linyi People's Hospital (Linyi, China) and was performed in accordance with the National Institutes of Health Guide for the Use of Laboratory Animals. A total of 30 pregnant female Sprague-Dawley rats from Guangdong Medical Laboratory Animal Center (Foshan, China), were used in the present study. The animals were housed in individual cages at $\sim 22 \pm 1^{\circ} \mathrm{C}$, and had access to water and food ad libitum. The rats were observed closely for the day of birth [postnatal day 0 (P0)]. The rat pups had access to water ad libitum and were maintained under a 12-h light/dark cycle at $\sim 22 \pm 1^{\circ} \mathrm{C}$. The treatment group rat pups received CAPE $(10,20$ or $40 \mathrm{mg} / \mathrm{kg}$ body weight) orally each day along with standard diet from P1 to P15.

Chemicals and antibodies. Sevoflurane and CAPE were purchased from Sigma-Aldrich (St. Louis, MO, USA). Fluoro-Jade C (0.001\%) was obtained from EMD Millipore (Billerica, MA, USA). Primary antibodies against activated caspase-3 (cat. no. sc-7149), -8 (cat. no. sc-56070), -9 (cat. no. sc-7885), B cell CCL/lymphoma 2 (Bcl-2; cat. no. 509), Bcl-2-associated agonist of cell death (Bad; cat. no. sc-8044), Bcl-2-like 1 (Bcl-xL; cat. no. sc-7195), Bcl-2-associated X protein (Bax; cat. no. sc-493), phosphorylated (p)-Bad (cat. no. sc-101640), $\beta$-actin (cat. no. sc-69879; Santa Cruz Biotechnology, Inc., Dallas, TX, USA), Akt (cat. no. 2920), p-Akt (cat. no. 4060), glycogen synthase kinase $3 \beta$ (GSK3 $\beta$; cat. no. 9315), p-GSK3 $\beta$ (cat. no. 9323), phosphatase and tensin homolog (PTEN; cat. no. 9556), JNK (cat. no. 9252), p-JNK (cat. no. 9255), p-c-Jun (cat. no. 2361), ERK1/2 (cat. no. 4615), p-ERK1/2 (cat. no. 4377), p38 (cat. no. 9228) and p-p38 (cat. no. 9215; Cell Signaling Technology, Inc., Danvers, MA, USA) were used in the current study. All chemicals used in this study were of analytical grade and procured from Sigma-Aldrich unless specified.

Anesthesia exposure. At P7, groups of rat pups were exposed to sevoflurane $(2.9 \%)$. The pups were retained in a humid chamber with total gas flow $2 \mathrm{l} / \mathrm{min}$, using $25 \% \mathrm{O}_{2}$ as the carrier. Anesthetic agent fractions and $\mathrm{O}_{2}$ were measured using a Capnomac Ultima gas analysis system (GE Healthcare Life Sciences, Chalfont, UK). During anesthetic exposure, the pups were placed on a warm mat at $38 \pm 1^{\circ} \mathrm{C}$. Neonatal rats were assigned to receive $2.9 \%$ sevoflurane for $6 \mathrm{~h}$ in $30 \% \mathrm{O}_{2}$ (38). On P7 the rats were administered with CAPE (10, 20 or $40 \mathrm{mg} / \mathrm{kg}$ body weight) $1 \mathrm{~h}$ prior to sevoflurane exposure. The control group received no anesthesia or CAPE. The anesthetic control group received only anesthesia and were not treated with CAPE. At the end of the study period, the animals ( $n=6$ per group) were anesthetized with sodium thiopenthal (100 mg/kg; Sigma-Aldrich) and were sacrificed after 25-30 min of thiopental injection. Samples of hippocampal tissue were excised for analysis of apoptosis by terminal deoxynucleotidyl transferase dUTP nick-end labeling (TUNEL) assay, and protein expression by western blotting.

Measurement of plasma 100 calcium binding protein $\beta$ (S100 $\beta$ ) by enzyme-linked immunosorbent assay (ELISA). The S100 family of dimeric cytosolic calcium binding proteins are expressed in astroglial and Schwann cells. The $\beta$ isomer of S100 is released into the extracellular space upon tissue injury and enters the serum through the blood brain barrier following mild brain injury, trauma, ischemia, hypoxia and exposure to neurotoxins (39). The levels of plasma $S 100 \beta$ in neonatal rats were evaluated using a Sangtec 100 ELISA kit (DiaSorin S.P.A., Gerenzano, Italy) according to the manufacturer's instructions. Briefly, $50 \mu 1$ plasma from each pup was added to a well of the 96-well plate and mixed with $150 \mu \mathrm{l}$ tracer from the kit, and incubated for $\sim 2 \mathrm{~h}$. Following incubation, 3,3',5,5'tetramethylbenzidine substrate and stop solution were added and the solution was mixed well. The optical density was measured at $450 \mathrm{~nm}$ using a Bio-Rad iMark microplate absorbance reader (Bio-Rad Laboratories, Inc., Hercules, CA, USA) (40).

Measurement of apoptosis by TUNEL assay. TUNEL assay was performed to assess neuronal apoptosis, as described previously by Li et al (6). Briefly, P7 rat pups exposed to sevoflurane were sacrificed and the brain tissues were excised. The sections were immersed in $10 \%$ buffered formalin for $15 \mathrm{~min}$ at room temperature. Tissues were post-fixed for $48 \mathrm{~h}$ at $4^{\circ} \mathrm{C}$, embedded in paraffin and sections $(5 \mu \mathrm{m})$ were used for the assay. A TUNEL fluorescent assay was performed using the fluorometric TUNEL system kit (Promega Corporation, Madision, WI, USA). The brain tissue slides were protected from direct light and the nuclei were stained using $2 \mu \mathrm{g} / \mathrm{ml}$ Hoechst for $10 \mathrm{~min}$. TUNEL-positive cells in the hippocampal dentate gyrus (DG), CA1 and CA3 regions 
were analyzed in 10 fields using the NIS-Elements BR image processing and analysis software (Nikon Corporation, Tokyo, Japan).

Immunohistochemical analysis of cleaved caspase-3. Apoptosis was analyzed by immunohistochemical analysis of cleaved caspase-3 levels, as previously described by Li et al (41). Briefly, the brain tissue sections were incubated with anti-cleaved caspase- 3 primary antibody at $4^{\circ} \mathrm{C}$ overnight, followed by biotin-conjugated secondary antibody treatment (1:200; cat. no. sc-2040; Santa Cruz Biotechnology, Inc.) for $\sim 40 \mathrm{~min}$ at room temperature. The sections were subsequently incubated with avidin-biotinylated peroxidase complex (Vectastain ABC kit; Vector Laboratories, Inc., Burlingame, CA, USA) for $40 \mathrm{~min}$ then stained with 3,3'-diaminobenzidine (Vector Laboratories, Inc.). The sections were observed using an IX70 microscope (Olympus Corporation, Tokyo, Japan) with 6 randomly chosen fields imaged per slide.

Immunoblotting. Hippocampi were isolated from the rat pups immediately following exposure to sevoflurane then used for western blotting as described previously $(5,6)$. The protein concentrations within the samples were determined using bicinchoninic acid protein assay (Bio-Rad Laboratories, Inc.). Protein samples $(60 \mu \mathrm{g})$ were separated by SDS-PAGE and electrotransferred to nitrocellulose membranes, then incubated with primary antibodies $(1: 1,000)$. The positive reactive bands were detected by Amersham ECL enhanced chemiluminescence western blotting detection kit (GE Healthcare Life Sciences). The blots were scanned using Image Master II scanner (GE Healthcare Life Sciences) and densities analyzed using Image Quant TL software (version 2003.03; GE Healthcare Life Sciences). The band densities were normalized to those of $\beta$-actin using anti- $\beta$-actin antibody. Western blotting was repeated six times for quantification.

Statistical analysis. The experimental data are presented as the mean \pm standard deviation, obtained from three or six individual experiments. The values were subjected to one-way analysis of variance followed by post-hoc Duncan's multiple range test using SPSS software (version 21.0; IBM SPSS, Armonk, NY, USA). P<0.05 was considered to indicate a statistically significant difference.

\section{Results}

CAPE inhibits plasma S100 $\beta$ levels. Previous studies have demonstrated that neuroapoptosis of the neonatal brain increases following exposure to inhaled or intravenous anesthetic agents $(7,42,43)$. S100 $\beta$, the $\beta$ isomer of S100, has previously been identified as a valuable biomarker for detecting anesthetic-induced neurodegeneration $(40,44)$. In the present study sevoflurane $(2.9 \%)$ caused $\sim 4$-fold increase in $\mathrm{S} 100 \beta$ levels, however administration of CAPE caused a significant decrease in the plasma levels of S100 $\beta$ (Fig. 1). Supplementation with $40 \mathrm{mg}$ CAPE exerted a more potent effect on the plasma $\mathrm{S} 100 \beta$ levels than the lower doses $(\mathrm{P}<0.05)$.

CAPE effectively inhibits sevoflurane-induced neurodegeneration. Anesthetics have been shown to induce significant neuronal apoptosis in the developing brain $(4,7)$. In the current study, a $6 \mathrm{~h}$ exposure to sevoflurane increased the number of TUNEL-positive cells in the hippocampi of P7 rat pups; the increase was greatest in CA1, followed by DG then CA3 ( $\mathrm{P}<0.05$; Fig. 2). Furthermore, CAPE administration significantly decreased the number of TUNEL-positive cells in the hippocampi of the rat pups $(\mathrm{P}<0.05)$. The $\mathrm{CA} 1$ region of the hippocampus exhibited a higher number of apoptotic cells when compared with the DG and CA3 regions $(\mathrm{P}<0.05)$.

Activated caspase-3 is commonly used as a biomarker for anesthesia-induced apoptosis (7,45). Zheng et al (46) demonstrated significant neural degeneration in the hippocampus following exposure to $1 \%$ sevoflurane. Thus, the present study examined the number of caspase-positive cells following sevoflurane and CAPE exposure. Consistent with the S100 $\beta$ level results, sevoflurane exposure significantly increased the number of caspase-3-positive cells in the hippocampal regions of the neonatal rats compared with the control. Administration of CAPE (10, 20 or $40 \mathrm{mg}$ ) resulted in a significant decrease in the number of caspase-positive cells in a dose-dependent manner $(\mathrm{P}<0.05$; Fig. 3$)$.

Furthermore, after $6 \mathrm{~h}$ exposure to inhaled sevoflurane $(2.9 \%)$, the expression levels of the pro-apoptotic proteins, caspase-3, -8 and -9 , were significantly upregulated compared with control levels $(\mathrm{P}<0.05$; Fig. 4$)$, as demonstrated by western blot analysis. Compared with those of the control, the expression levels of Bad and Bax were significantly increased by sevoflurane $(\mathrm{P}<0.05)$. Sevoflurane reduced the expression levels of anti-apoptotic proteins, Bcl-2 and Bcl-xL, when compared with control levels ( $\mathrm{P}<0.05$; Fig. 4). CAPE treatment significantly downregulated the expression of caspases, and Bax and Bad compared with sevoflurane treatment $(\mathrm{P}<0.05)$, whereas the expression levels of $\mathrm{Bcl}-\mathrm{xL}$ and $\mathrm{Bcl}-2$ were increased $(\mathrm{P}<0.05)$. This indicated that CAPE may exert its anti-apoptotic effects by modulating the expression of caspases and apoptotic pathway proteins.

Neuroprotection by CAPE involves the JNK, ERK and p38 signaling pathways. To further investigate the molecular mechanisms associated with neuroprotection by CAPE, the expression of MAPK family proteins (JNK, ERK1/2 and p38 MAPK) were examined. Previous studies have demonstrated that JNK, ERK1/2 and p38 are involved in dexmedetomidine-induced neuroprotection $(41,47,48)$. The present study demonstrated that the levels of p-JNK and p-p38 kinases were significantly upregulated following sevoflurane exposure compared with control $(\mathrm{P}<0.05$; Fig. 5). However, the sevoflurane-increased ERK1/2 levels were not as high as the JNK levels. In addition to the enhanced expression of JNK, the levels of p-c-Jun were increased compared with the control $(\mathrm{P}<0.05)$. CAPE significantly downregulated the expression of $\mathrm{p}-\mathrm{JNK}, \mathrm{p}-\mathrm{ERK}$ and $\mathrm{p}-\mathrm{p} 38$, and reduced the expression of p-c-Jun compared with the sevoflurane group $(\mathrm{P}<0.05)$. Furthermore, CAPE significantly downregulated the expression levels of total JNK, ERK1/2 and p38 when compared with sevoflurane treatment. However, comparatively, CAPE exhibited a less potent effect on ERK1/2 and p-ERK1/2 levels compared with JNK and $\mathrm{p} 38$. These results indicated that the MAPK signaling pathway is involved in CAPE-mediated neuroprotection. 


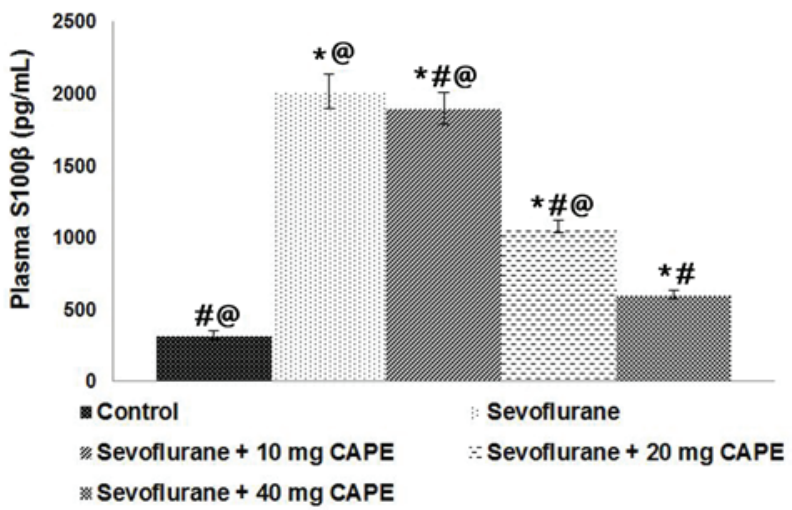

Figure 1. Plasma S100 $\beta$ levels in mice at post-natal day 7 following exposure to sevoflurane. CAPE effectively reduced the plasma S100 $\beta$ levels. Values are presented as the mean \pm standard deviation $(\mathrm{n}=6)$. ${ }^{*} \mathrm{P}<0.05$ vs. control; ${ }^{*} \mathrm{P}<0.05$ vs. sevoflurane; ${ }^{\circledR} \mathrm{P}<0.05 \mathrm{vs} .40 \mathrm{mg}$ CAPE; as determined by one-way analysis of variance followed by Duncan's multiple range test. $\mathrm{S} 100 \beta, \mathrm{S} 100$ calcium binding protein $\beta$; CAPE, caffeic acid phenethyl ester.

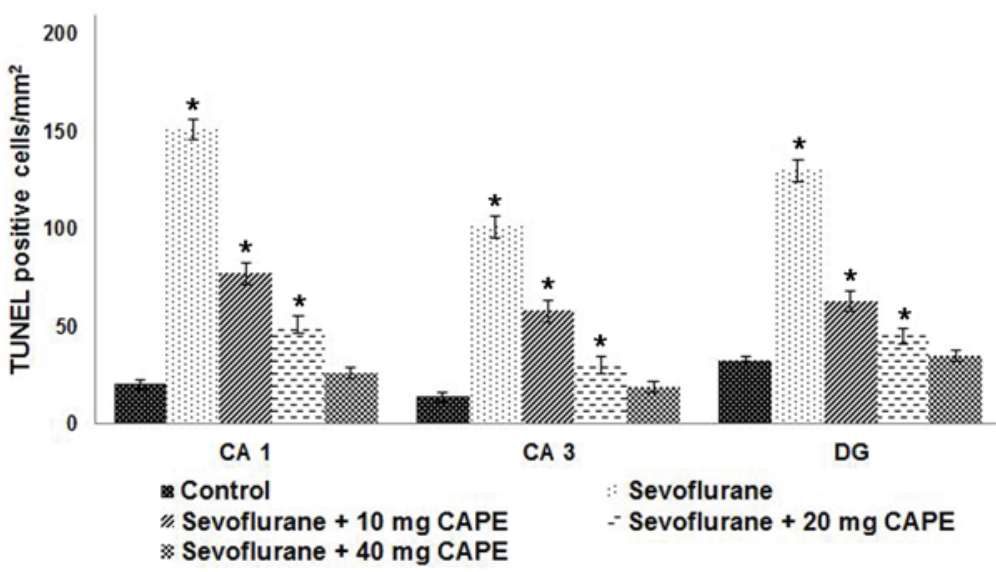

Figure 2. Effect of CAPE on sevoflurane-induced neuroapoptosis in rat pups on post-natal day 7. Sevoflurane induced robust neurodegeneration in the hippocampi of the rat pups. CAPE significantly inhibited neuroapotosis induced by the anesthetic. Values are presented as the mean \pm standard deviation ( $\mathrm{n}=6$ ). ${ }^{*} \mathrm{P}<0.05$ vs. control as determined by one-way analysis of variance. TUNEL, terminal deoxynucleotidyl transferase dUTP nick-end labeling; DG, dentate gyrus; CAPE, caffeic acid phenethyl ester.

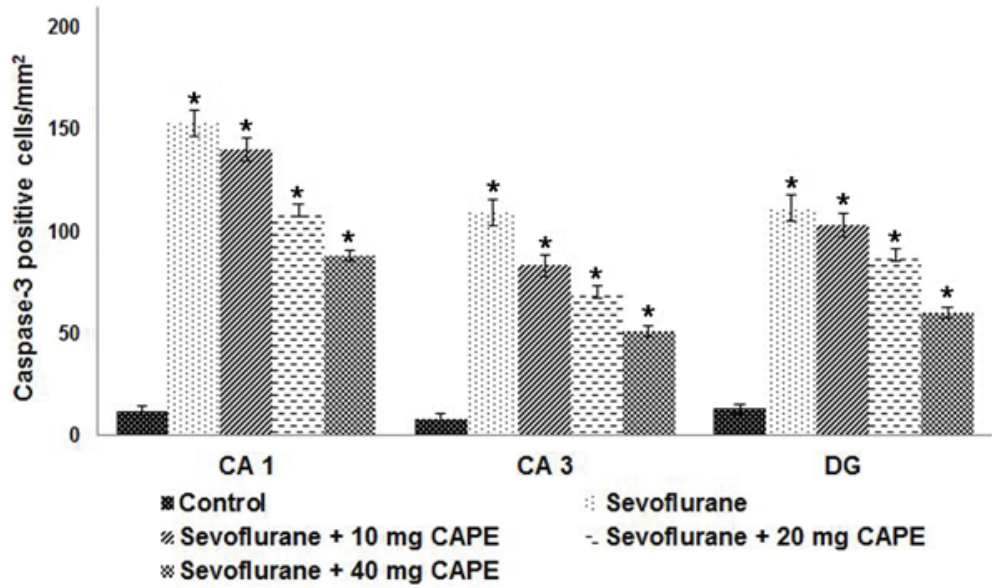

Figure 3. CAPE reduces the intensive apoptotic neurodegeneration due to neonatal anesthesia. Exposure to sevoflurane for $6 \mathrm{~h}$ caused multi-fold increase in the level of cleaved capase- 3 expression. CAPE treatment significantly reduced caspase- 3 positive cell counts. Values are presented as the mean \pm standard deviation $(n=6) . ~ P<0.05$ vs. control as determined by one-way analysis of variance. DG, dentate gyrus; CAPE, caffeic acid phenethyl ester.

PI3K/Akt signaling pathway is involved in neuroprotection of neonatal brain cells by CAPE. The mechanisms involved in inhalational anesthetic-induced neuronal apoptosis in neonatal brains have been widely investigated. The current study evaluated the affect of sevoflurane on PI3K/Akt signaling pathway proteins. The PI3K/Akt/mechanistic target 
A

B
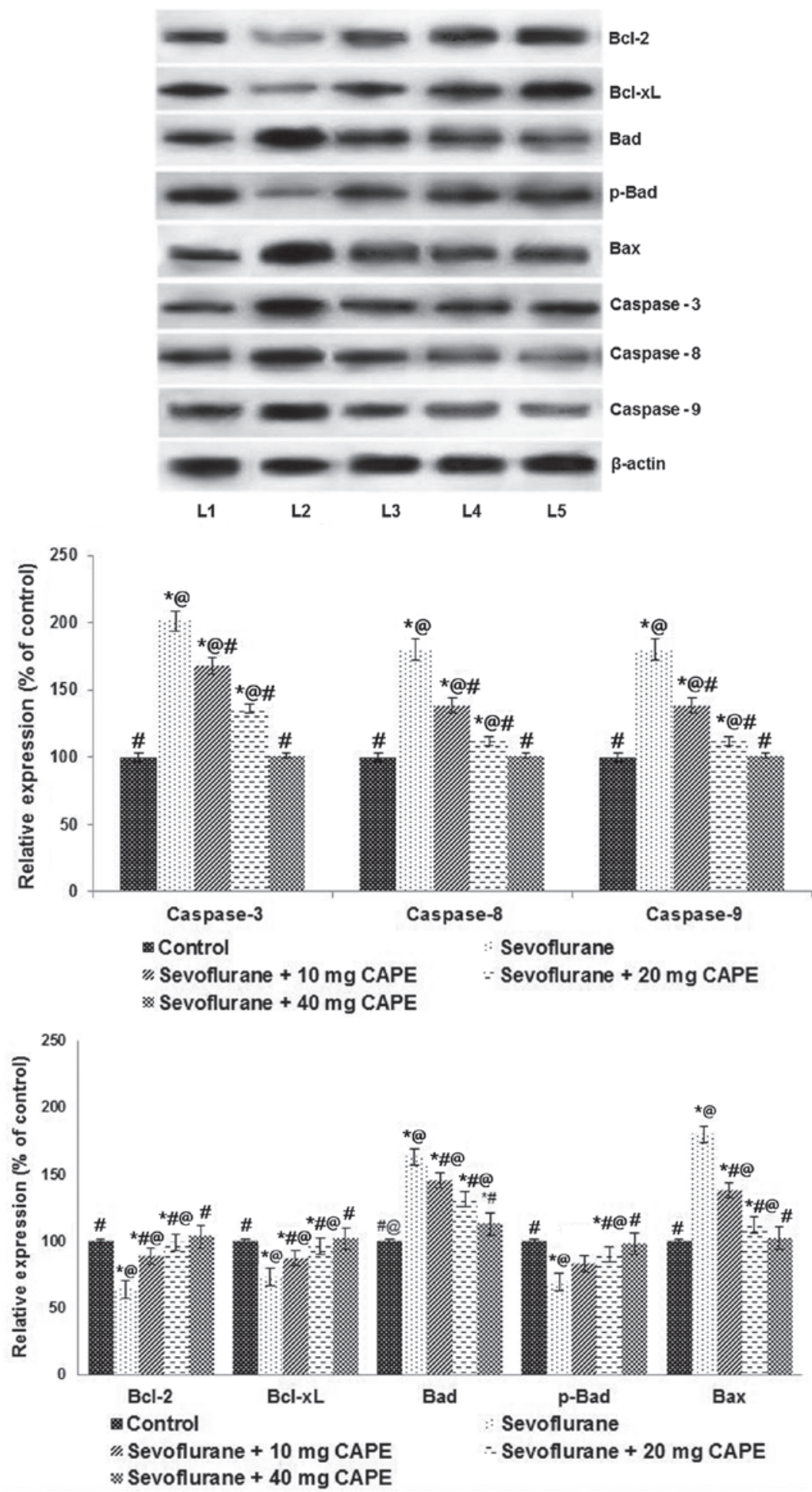

Figure 4. CAPE regulates the expression of apoptotic pathway proteins following sevoflurane anesthesia. (A) Sevoflurane significantly enhanced the expression levels of caspases and the pro-apoptotic proteins, Bax and Bad. CAPE supplementation caused marked protein expression regulation. Lane 1, control; lane 2, sevoflurane; lane 3, sevoflurane + $10 \mathrm{mg}$ CAPE; lane 4, sevoflurane + $20 \mathrm{mg}$ CAPE; lane 5, sevoflurane + $40 \mathrm{mg}$ CAPE. (B) Relative expression levels of the proteins. Values are presented as the mean \pm standard deviation $(\mathrm{n}=3)$. ${ }^{*} \mathrm{P}<0.05$ vs. control; ${ }^{*} \mathrm{P}<0.05$ vs. sevoflurane; ${ }^{\circledR} \mathrm{P}<0.05$ vs. $40 \mathrm{mg} \mathrm{CAPE}$; as determined by one-way analysis of variance followed by Duncan's multiple range test. Bcl-2, B cell CCL/lymphoma 2; Bcl-xL, Bcl-2-like 1; p-Bad, phosphorylated Bcl-2-associated agonist of cell death; Bax, Bcl-2-associated X protein; CAPE, caffeic acid phenethyl ester.

of rapamycin signaling pathway is important for regulating the cell cycle, and previous reports have demonstrated that $\mathrm{InsP}_{3} \mathrm{Rs}$ and variations in intracellular calcium homeostasis are involved in anesthesia-induced neurodegeneration $(29,49)$.
Sevoflurane exposure significantly reduced the levels of Akt and p-Akt $(\mathrm{P}<0.05$; Fig. 6). Additionally, a significant decrease in the expression levels of GSK3 $\beta$ and p-GSK3 $\beta$ levels were observed following $6 \mathrm{~h}$ of exposure to sevoflurane compared 
A

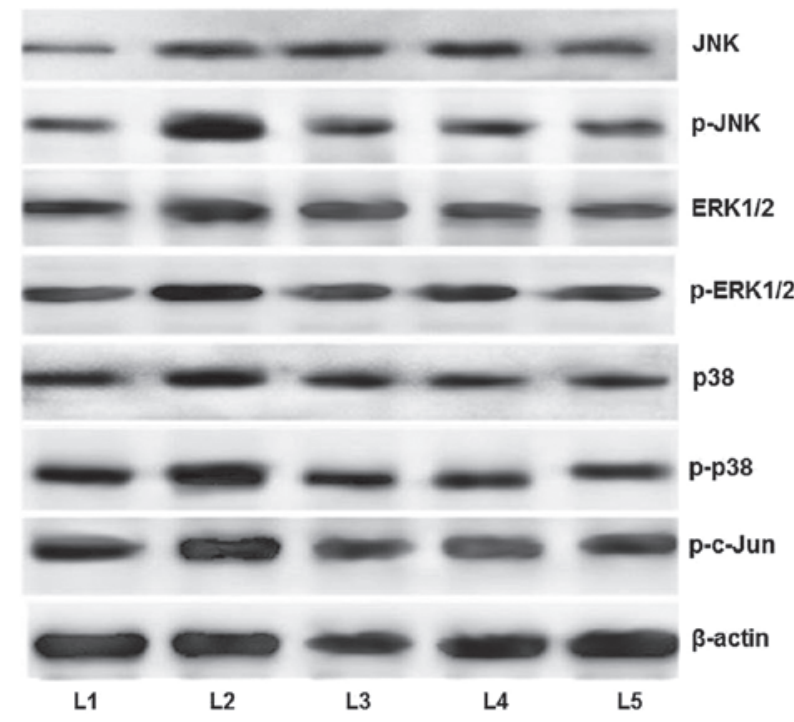

B

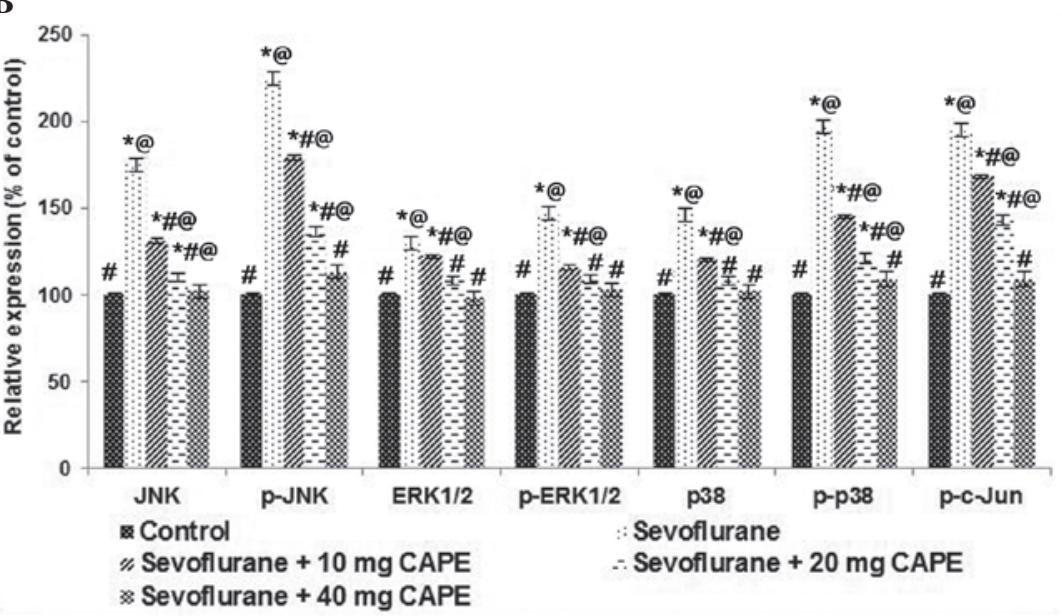

Figure 5. CAPE regulates the expression of mitogen-activated protein kinases. (A) Sevoflurane-induced significantly enhanced protein expression levels were modulated by CAPE in a dose-dependent manner. Lane 1, control; lane 2, sevoflurane; lane 3, sevoflurane + $10 \mathrm{mg}$ CAPE; lane 4, sevoflurane + $20 \mathrm{mg}$ CAPE; lane 5, sevoflurane $+40 \mathrm{mg}$ CAPE. (B) Relative expression levels of the proteins. Values are presented as the mean \pm standard deviation ( $\mathrm{n}=6$ ). ${ }^{*} \mathrm{P}<0.05$ vs. control; ${ }^{\sharp} \mathrm{P}<0.05$ vs. sevoflurane; ${ }^{\circledR} \mathrm{P}<0.05$ vs. $40 \mathrm{mg}$ CAPE; as determined by one-way analysis of variance followed by Duncan's multiple range test. JNK, c-Jun N-terminal kinase; p, phosphorylated; ERK, extracellular signal-regulated kinase; CAPE, caffeic acid phenethyl ester.

with the control $(\mathrm{P}<0.05$; Fig. 6). CAPE supplementation downregulated the PI3K/Akt signaling pathway, as demonstrated by a significant increase in Akt expression levels and enhanced GSK3 $\beta$ expression $(\mathrm{P}<0.05)$. Additionally, PTEN expression levels were observed to be enhanced by CAPE treatment compared with groups exposed to sevoflurane only $(\mathrm{P}<0.05)$, suggesting that activation of the PI3K/Akt pathway is involved in neuroprotection.

\section{Discussion}

Growing experimental data have reported that widespread neuroapoptosis occurs in developing brain cells following early exposure to commonly used general anesthetics $(10,11,50,51)$. Volatile anesthetic, sevoflurane, has previously been demonstrated to induce apoptotic neurodegeneration in the developing rat brain and to cause persistent learning/memory deficits $(12,52)$.

Cell death by apoptosis is a vital aspect of normal brain maturation that leads to the elimination of $50-70 \%$ of progenitor cells and neurons during development $(53,54)$. However, neuronal apoptosis exceeding this natural apoptotic rate is triggered by various pathologic conditions, including hypoxia, ischemia or prolonged anesthetic exposure $(55,56)$. Accordingly, the current study examined the level of neuronal apoptosis in the hippocampi of P7 rats exposed to $6 \mathrm{~h}$ of sevoflurane anesthesia.

The expression of cleaved caspase-3 expression, a validated marker of cell death, was measured to detect apoptosis. Caspase-3, an aspartate-specific cysteine protease, is an important executioner protein of the apoptosis pathway (57). In the present study, immunohistochemistry and western blot analysis demonstrated that sevoflurane exposure leads to a increase in the protein expression of cleaved caspase- 3 in the hippocampus. Neuronal apoptosis was more severe in the CA1 region than the CA3 and DG regions, as detected by immunohistochemistry and TUNEL assay. These findings were consistent with those of previous studies $(12,13,38)$. Furthermore, the expression levels of initiator caspases (caspase- 8 
A

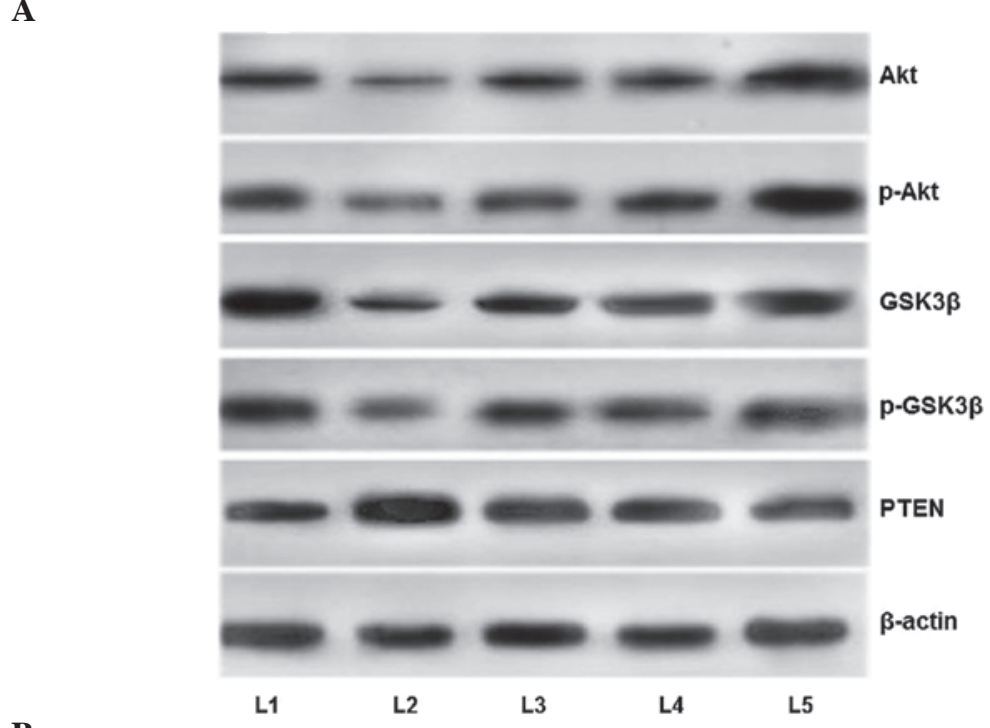

B

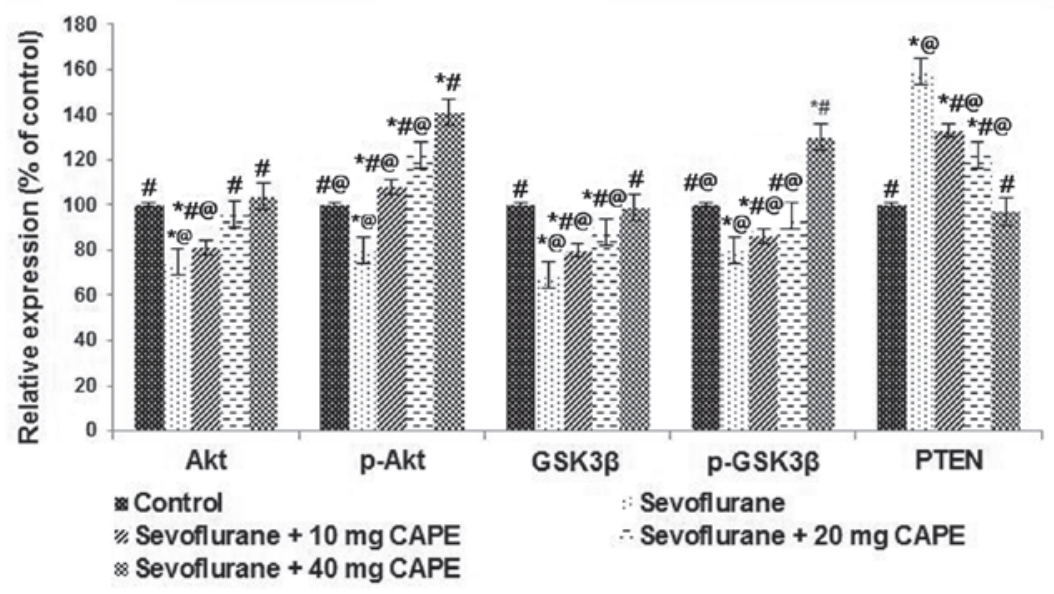

Figure 6. CAPE may activate the PI3K/Akt signaling pathway following sevoflurane exposure. (A) Sevoflurane at $2.9 \%$ significantly downregulated the PI3K/Akt signaling pathway. CAPE treatment activated the PI3K/Akt signaling pathway. Lane 1, control; lane 2, sevoflurane; lane 3, sevoflurane $+10 \mathrm{mg}$ CAPE; lane 4, sevoflurane + $20 \mathrm{mg}$ CAPE; lane 5, sevoflurane + $40 \mathrm{mg}$ CAPE. (B) Values are presented as the mean \pm standard deviation ( $\mathrm{n}=6$ ). ${ }^{*} \mathrm{P}<0.05$ vs. control; " $\mathrm{P}<0.05$ vs. sevoflurane; ${ }^{\circledR} \mathrm{P}<0.05$ vs. $40 \mathrm{mg}$ CAPE; as determined by one-way analysis of variance followed by Duncan's multiple range test. $\mathrm{p}$, phosphorylated; GSK3 $\beta$, glycogen synthase kinase 3 $\beta$; PTEN, phosphatase and tensin homolog; PI3K, phosphoinositide 3-kinase CAPE, caffeic acid phenethyl ester.

and -9) were observed to be enhanced by sevoflurane exposure. Previous studies have demonstrated an association between anesthetic-induced apoptosis and elevated plasma S100 $\beta$ levels, which could potentially be used as a neurodegenerative biomarker for brain damage following various types of stress $(40,44)$. In accordance with previous reports, sevoflurane exposure caused a significant increase in plasma S100 $\beta$ levels. Downregulation of the apoptotic cell counts and the expression of caspases (caspase- $3,-8$ and -9 ) by CAPE, suggests that CAPE suppresses sevoflurane-induced apoptosis.

The balance between the anti-apoptotic (Bcl-2 and $\mathrm{Bcl}-\mathrm{xL})$ and pro-apoptotic (Bad and Bax) Bcl-2 family proteins regulates cell survival and death (58). Bad is activated through phosphorylation (59) by proto-oncogene proteins $c$-Akt that subsequently leads to the binding of Bad with 14-3-3, a cytosolic protein, and causes the release of anti-apoptotic protein, $\mathrm{Bcl}-\mathrm{xL}$. Bcl-xL binds to Bax and consequently inhibits apoptosis $(60,61)$. Thus, Bcl-xL and Bcl-2 block Bax translocation to the mitochondria and maintain the mitochondrial membrane potential to prevent subsequent apoptosis (61). The enhanced expression of Bad and Bax following sevoflurane exposure observed in the current study suggests that the apoptosis rate is elevated by sevoflurane, which correlates with suppression of Bcl-xl and Bcl-2. Bcl-xL, expressed extensively in the central nervous system (CNS), enriches cell survival by maintaining mitochondrial membrane integrity and reducing cytochrome complex release (58). An anesthesia combination containing nitrous oxide, isoflurane and midazolam has previously been reported to downregulate $\mathrm{Bcl}-\mathrm{xL}$, leading to neurotoxicity (62). In the present study, CAPE, at 10-, 20- and 40-mg doses, increased neuronal cell survival, which was demonstrated by the upregulation of anti-apoptotic proteins and significant inhibition of Bax and Bad expression levels.

The PI3K/Akt intracellular signaling pathway is associated with cellular quiescence, proliferation, cell survival and cancer. Activated PI3K phosphorylates and activates Akt, localizing it to the plasma membrane (63). The PI3K/Akt signaling pathway is crucial in the decision between cell proliferation and renewal, as opposed to differentiation and quiescence. The pathway is antagonized by various factors, including 
PTEN (64) GSK3 $\beta$ (63) and homeobox gene Hb9 (65). Upon activation, Akt inhibits apoptosis via phosphorylation of Bad and GSK3 $\beta(66,67)$. Previous reports suggest a potential link between Akt and JNK signaling, and Akt signaling is reported to be involved in the apoptotic effect of JNK (68). Furthermore, a selective JNK inhibitor, SP600125 (67) was demonstrated to exhibit neuroprotective effects $(69,70)$.

In the present study, sevoflurane exposure inhibited the activation of Akt and upregulated the expression levels of PTEN. In addition, sevoflurane reduced the level of p-GSK3 $\beta$ and p-Akt, which promote the apoptosis of neuronal cells. CAPE potentially activates the PI3K/Akt signaling pathway by significantly increasing the expression and phosphorylation of Akt and GSK3 $\beta$. Silencing the $\operatorname{InsP}_{3} \mathrm{R}$ was previously demonstrated to inhibit isoflurane-induced neuroapoptosis (29), potentially contributing to the inhibition of neuroapoptosis by this mechanism. However, this hypothesis requires further validation.

Furthermore, PTEN levels were suppressed by CAPE, contributing to the effect of CAPE on the PI3K/Akt signaling cascade. PTEN inhibition has previously been reported to promote neuroprotection following CNS injury (71). Thus, inhibition of PTEN expression, which was demonstrated in the current study, may also contribute to the neuroprotective effects of CAPE.

JNK signaling is associated with neuronal apoptosis activated by various stimuli that cause brain injury, including ischemia/reperfusion and ethanol (72-74). Previous studies have demonstrated that the JNK signaling pathway is activated in isoflurane-induced neuronal apoptosis (6). SP600125, a JNK inhibitor, prevented the phosphorylation of c-Jun, a substrate of $\mathrm{JNK}$, and neuroapoptosis induced by isoflurane $(6,75)$. In the present study, sevoflurane increased the levels of p-JNK and p-c-Jun in the hippocampi of P7 rats, suggesting that the JNK signaling pathway is activated in sevoflurane-induced neuronal apoptosis. Expression of ERK1/2 and its phosphorylated forms, was also enhanced marginally by sevoflurane. CAPE downregulated the expression levels of JNK and ERK1/2 in a dose-dependent manner, indicating that the effects of CAPE may involve the JNK and ERK signaling pathways.

Previous reports have demonstrated that the $\mathrm{p} 38$ MAPK signaling pathway is involved in anesthetic-induced neurodegeneration (76) and that p38 is enhanced in isoflurane-induced neuronal apoptosis (75). In the current study, CAPE prevented the sevoflurane-induced increase in p-p38 expression levels, suggesting that the $\mathrm{p} 38$ signaling pathway is involved in the neuroprotective effect of CAPE. Treatment with dexmedetomidine and p38 MAPK inhibitor, SB203580 was previously demonstrated to decrease the expression level of p-p38, suggesting that the p38 signaling pathway is also involved in the neuroprotective effects of dexmedetomidine (75).

In conclusion, the observations of the current study indicate that CAPE inhibits sevoflurane-induced neuronal apoptosis in the neonatal rat brain via modulating the expression of caspases and regulating the critical pathways involved in neuronal apoptosis, including the JNK/ERK/p38 MAPK and PI3K/Akt signaling pathways. Thus, CAPE may be a potential candidate for reducing anesthetic-induced neurotoxicity. However, further investigations using specific JNK/ERK and Akt, and studies on the apoptotic pathway inhibitors are required to assess the neuroprotective effects of CAPE.

\section{References}

1. Istaphanous GK and Loepke AW: General anesthetics and the developing brain. Curr Opin Anesthesiol 22: 368-373, 2009.

2. Patel SS and Goa KL: Sevoflurane: A review of its pharmacodynamic and pharmacokinetic properties and its clinical use in general anaesthesia. Drugs 51: 658-700, 1996.

3. Johnson SA, Young C and Olney JW: Isoflurane-induced neuroapoptosis in the developing brain of nonhypoglycemic mice. J Neurosurg Anesthesiol 20: 21-28, 2008.

4. Brambrink AM, Evers AS, Avidan MS, Farber NB Smith DJ, Zhang X, Dissen GA, Creeley CE and Olney JW: Isoflurane-induced neuroapoptosis in theneonatal rhesus macaque brain. Anesthesiology 112: 834-841, 2010.

5. Li Y, Liu C, Zhao Y, Hu K, Zhang J, Zeng M, Luo T, Jiang W and Wang H: Sevoflurane induces short-term changes in proteins in the cerebral cortices of developing rats. Acta Anaesthesiol Scand 57: 380-390, 2013.

6. Li Y, Wang F, Liu C, Zeng M, Han X, Luo T, Jiang W, Xu J and Wang H: JNK pathway may be involved in isoflurane-induced apoptosis in the hippocampi of neonatal rats. Neurosci Lett 545: 17-22, 2013.

7. Jevtovic-Todorovic V, Hartman RE, Izumi Y, Benshoff ND, Dikranian K, Zorum-ski CF, Olney JW and Wozniak DF: Early exposure to common anesthetic agents causes widespread neurodegeneration in the developing rat brain and persistent learning deficits. J Neurosci 23: 876-882, 2003.

8. Zhu C, Gao J, Karlsson N, Li Q, Zhang Y, Huang Z, Li H, Kuhn HG and Blomgren K: Isoflurane anesthesia induced persistent, progressive memory impairment, caused a loss of neural stem cells and reduced neurogenesis in young, but not adult, rodents. J Cereb Blood Flow Metab 30: 1017-1030, 2010.

9. Stratmann G, Sall JW, May LD, Bell JS, Magnusson KR, Rau V, Visrodia KH, Alvi RS, Ku B, Lee MT and Dai R: Isoflurane differentially affects neurogenesis and long-term neurocognitive function in 60 day-old and 7 day-old rats. Anesthesiology 110: 834-848, 2009.

10. DiMaggio C, Sun LS and Li G: Early childhood exposure to anesthesia and risk of developmental and behavioral disorders in a sibling birth cohort. Anesth Analg 113: 1143-1151, 2011.

11. Ing C, DiMaggio C, Whitehouse A, Hegarty MK, Brady J, von Ungern-Sternberg BS, Davidson A, Wood AJ, Li G and Sun LS: Long-term differences in language and cognitive function after childhood exposure to anesthesia. Pediatrics 130: e476-e485, 2012

12. Satomoto M, Satoh Y, Terui K, Miyao H, Takishima K, Ito M and Imaki J: Neonatal exposure to sevoflurane induces abnormal social behaviors and deficits in fear conditioning in mice. Anesthesiology 110: 628-637, 2009.

13. Kodama M, Satoh Y, Otsubo Y, Araki Y, Yonamine R, Masui K and Kazama T: Neonatal desflurane exposure induces more robust neuroapoptosis than do isoflurane and sevoflurane and impairs working memory. Anesthesiology 115: 979-991, 2011.

14. Shih J, May LD, Gonzalez HE, Lee EW, Alvi RS, Sall JW, Rau V, Bickler PE, Lalchandani GR, Yusupova M, et al: Delayed environmental enrichment reverses sevoflurane-induced memory impairment in rats. Anesthesiology 116: 586-602, 2012.

15. Wei HF, Liang G, Yang H, Wang Q, Hawkins B, Madesh M, Wang S and Eckenhoff RG: The common inhalational anesthetic isoflurane induces apoptosis via activation of inositol 1,4,5-trisphosphate receptors. Anesthesiology 108: 251-260, 2008.

16. Lunardi N, Ori C, Erisir A and Jevtovic-Todorovic V: General anesthesia causes long-lasting disturbances in the ultrastructural properties of developing synapses in young rats. Neurotox Res 17: 179-188, 2010.

17. Zhao X, Yang Z, Liang G, Wu Z, Peng Y, Joseph DJ, Inan S and Wei H: Dual effects of isoflurane on proliferation, differentiation and survival in human neuroprogenitor cells. Anesthesiology 118: 537-549, 2013.

18. Soriano SG, Liu Q, Li J, Liu JR, Han XH, Kanter JL, Bajic D and Ibla JC: Ketamine activates cell cycle signaling and apoptosis in the neonatal rat brain. Anesthesiology 112: 1155-1163, 2010.

19. Sinner B, Friedrich O, Zink W, Zausig Y and Graf BM: The toxic effects of $s(+)$-ketamine on differentiating neurons in vitro as a consequence of suppressed neuronal $\mathrm{Ca}^{2+}$ oscillations. Anesth Analg 113: 1161-1169, 2011.

20. Zhao YL, Xiang Q, Shi QY, Li SY, Tan L, Wang JT, Jin XG and Luo AL: GABAergic excitotoxicity injury of the immature hippocampal pyramidal neurons' exposure to isoflurane. Anesth Analg 113: 1152-1160, 2011. 
21. Brambrink AM, Evers AS, Avidan MS, Farber NB Smith DJ, Martin LD, Dissen GA, Creeley CE and Olney JW: Ketamine-induced neuroapoptosis in the fetal and neonatal rhesus macaque brain. Anesthesiology 116: 372-384, 2012.

22. Istaphanous GK, Ward CG, Nan X, Hughes EA, Mccann JC McAuliffe JJ, Danzer SC and Loepke AW: Characterization and quantification of isoflurane-induced developmental apoptotic cell death in mouse cerebral cortex. Anesth Analg 116: 845-854, 2013.

23. Mousa $\mathrm{A}$ and Bakhiet $\mathrm{M}$ : Role of cytokine signaling during nervous system development. Int J Mol Sci 14: 13931-13957, 2013.

24. Harper SJ and Wilkie N: MAPKs: New targets for neurodegeneration. Expert Opin Ther Targets 7: 187-200, 2003

25. Kaminska B, Gozdz A, Zawadzka M, Ellert-Miklaszewska A and Lipko M: MAPK signal transduction underlying brain inflammation and gliosis as therapeutic target. Anat Rec (Hoboken) 292: 1902-1913, 2009.

26. Wang WY, Yang R, Hu SF, Wang H, Ma ZW and Lu Y: $\mathrm{N}$-stearoyl-l-tyrosine ameliorates sevoflurane induced neuroapoptosis via MEK/ERK1/2MAPK signaling pathway in the developing brain. Neurosci Lett 541: 167-172, 2013.

27. Sanders RD, Sun P, Patel S, Li M, Maze M and Ma D: Dexmedetomidine provides cortical neuroprotection: Impact on anaesthetic-induced neuroapoptosisin the rat developing brain Acta Anaesthesiol Scand 54: 710-716, 2010.

28. Li Y, Zeng M, Chen W, Liu C, Wang F, Han X, Zuo Z and Peng S Dexmedetomidine reduces isoflurane-induced neuroapoptosis partly by pre-serving PI3K/Akt pathway in the hippocampus of neonatal rats. PLoS One 9: e93639, 2014

29. Zhao Y, Liang G, Chen Q, Joseph DJ, Meng Q, Eckenhoff RG Eckenhoff MF and Wei H: Anesthetic-induced neurodegeneration mediated via inositol 1,4,5-trisphosphate receptors J Pharmacol Exp Ther 333: 14-22, 2010.

30. BaiT,Dong DS and Pei L: Resveratrolmitigates isoflurane-induced neuroapoptosis by inhibiting the activation of the Akt-regulated mitochondrial apoptotic signaling pathway. Int J Mol Med 32: 819-826, 2013.

31. Demestre M, Messerli SM, Celli N, Shahhossini M, Kluwe L, Mautner V and Maruta H: CAPE (caffeic acid phenethy ester)-based propolis extract (Bio 30) suppresses the growth of human neurofibromatosis (NF) tumor xenografts in mice. Phytother Res 23: 226-230, 2009.

32. Natarajan K, Singh S, Burke TR Jr, Grunberger D and Aggarwal BB: Caffeic acid phenethyl ester is a potent and specific inhibitor of activation of nuclear transcription factor NF-kappaB. Proc Natl Acad Sci USA 93: 9090-9095, 1996.

33. Lin HP, Jiang SS and Chuu CP: Caffeic acid phenethyl ester causes p21 induction, Akt signaling reduction and growth inhibition in PC-3 human prostate cancer cells. PLoS One 7: e31286, 2012.

34. Orban Z, Mitsiades N, Burke TR, Tsokos M and Chrousos GP: Caffeic acid phenethyl ester induces leukocyte apoptosis, modulates nuclear factor-kappaB and suppresses acute inflammation. Neuroimmunomodulation 7: 99-105, 2000.

35. Irmak MK, Fadillioglu E, Sogut S, Erdogan H, Gulec M, Ozer M Yagmurca M and Gozukara ME: Effects of caffeic acid phenethyl ester and alpha-tocopherol on reperfusion injury in rat brain. Cell Biochem Funct 21: 283-289, 2003.

36. Altug ME, Serarslan Y, Bal R, Kontas T, Ekici F, Melek IM, Aslan H and Duman T: Caffeic acid phenethyl ester protects rabbit brains against permanent focal ischemia by antioxidant action: A biochemical and planimetric study. Brain Res 1201: 135-142, 2008

37. Kurauchi Y, Hisatsune A, Isohama Y, Mishima $\mathrm{S}$ and Katsuki $\mathrm{H}$ Caffeic acid phenethyl ester protects nigral dopaminergic neurons via dual mechanisms involving haem oxygenase-1 and brain-derived neurotrophic factor. Br J Pharmacol 166 $1151-1168,2012$

38. Istaphanous GK, Howard J, Nan X, Hughes EA, McCann JC, McAuliffe JJ, Danzer SC and Loepke AW: Comparison of the neuroapoptotic properties of equipotent anesthetic concentrations of desflurane, isoflurane, or sevoflurane in neonatal mice. Anesthesiology 114: 578-587, 2011.

39. Bloomfield SM, McKinney J, Smith L and Brisman J: Reliability of S100B in predicting severity of central nervous system injury. Neurocritical Care 6: 121-138, 2007

40. Wang S, Peretich K, Zhao Y, Liang G, Meng Q and Wei H: Anesthesia induced neurodegeneration in fetal rat brains. Pediatr Res 66: 435-440, 2009
41. Li B, Du T, Li H, Gu L, Zhang H, Huang J, Hertz L and Peng L: Signaling pathways for transactivation by dexmedetomidine of epidermal growth factor receptors in astrocytes and its paracrine effect on neurons. Br J Pharmacol 154: 191-203, 2008.

42. Pearn ML, Hu Y, Niesman IR, Patel HH, Drummond JC, Roth DM, Akassoglou K, Patel PM and Head BP: Propofol neurotoxicity is mediated by $\mathrm{p} 75$ neurotrophin receptor activation. Anesthesiology 116: 352-361, 2012.

43. Creeley C, Dikranian K, Dissen G, Martin L, Olney J and Brambrink A: Propofol induced apoptosis of neurones and oligodendrocytes in fetal and neonatal rhesus macaque brain. $\mathrm{Br}$ J Anaesth 110: i29-i38, 2013.

44. Liang G, Ward C, Peng J, Zhao Y, Huang B and Wei H: Isoflurane causes greater neurodegeneration than an equivalent exposure of sevoflurane in the developing brain of neonatal mice. Anesthesiology 112: 1325-1334, 2010 .

45. Dong Y, Zhang G, Zhang B, Moir RD, Xia W, Marcantonio ER, Culley DJ, Crosby G, Tanzi RE and Xie Z: The common inhalational anesthetic sevoflurane induces apoptosis and increases beta-amyloid protein levels. Arch Neurol 66: 620-631, 2009.

46. Zheng SQ, An LX, Cheng1 X and Wang YJ: Sevoflurane causes neuronal apoptosis and adaptability changes of neonatal rats. Acta Anaesthesiol Scand 57: 1167-1174, 2013.

47. Du T, Li B, Liu S, Zang P, Prevot V, Hertz L and Peng L: ERK phosphorylationin intact, adult brain by alpha(2)-adrenergic transactivation of EGF receptors. Neurochem Int 55: 593-600, 2009.

48. Zhang X, Wang J, Qian W, Zhao J, Sun L, Qian Y and Xiao H: Dexmedetomidine inhibits tumor necrosis factor-alpha and interleukin 6 inlipopolysaccharide-stimulated astrocytes by suppression of c-Jun N-terminalkinases. Inflammation 37 : 942-949, 2014.

49. Yang H, Liang G, Hawkins BJ, Madesh M, Pierwola A and Wei $\mathrm{H}$ : Inhalational anesthetics induce cell damage by disruption of intracellular calcium homeostasis with different potencies. Anesthesiology 109: 243-250, 2008.

50. Flick RP, Katusic SK, Colligan RC, Wilder RT, Voigt RG, Olson MD, Sprung J, Weaver AL, Schroeder DR and Warner DO: Cognitive and behavioral outcomes after early exposure to anesthesia and surgery. Pediatrics 128: e1053-e1061, 2011.

51. Bong CL, Allen JC and Kim JT: The effects of exposure to general anesthesia in infancy on academic performance at age 12. Anesth Analg 117: 1419-1428, 2013.

52. Fang F, Xue Z and Cang J: Sevoflurane exposure in 7 day-old rats affects neurogenesis, neurodegeneration and neurocognitive function. Neurosci Bull 28: 499-508, 2012.

53. Oppenheim RW: Cell death during development of the nervous system. Annu Rev Neurosci 14: 453-501, 1991.

54. Rakic S and Zecevic N: Programmed cell death in the developing human telencephalon. Eur J Neurosci 12: 2721-2734, 2000.

55. Blomgren K, Leist M and Groc L: Pathological apoptosis in the developing brain. Apoptosis 12: 993-1010, 2007.

56. Loepke AW and Soriano SG: An assessment of the effects of general anesthetics on developing brain structure and neurocognitive function. Anesth Analg 106: 1681-1707, 2008.

57. Gown AM and Willingham MC: Improved detection of apoptotic cells in archival paraffin sections: Immunohistochemistry using antibodies to cleaved caspase 3. J Histochem Cytochem 50: 449-454, 2002.

58. Zhao H, Yenari MA, Cheng D, Sapolsky RM and Steinberg GK: Bcl-2 overexpression protects against neuron loss within the ischemic margin following experimental stroke and inhibits cytochrome $\mathrm{c}$ translocation and caspase-3 activity. J Neurochem 85: 1026-1036, 2003

59. Chong ZZ, Li F and Maiese K: Oxidative stress in the brain: Novel cellular targets that govern survival during neurodegenerative disease. Prog Neurobiol 75: 207-246, 2005.

60. Hou J, Wang S, Shang YC, Chong ZZ and Maiese K: Erythropoietin employs cell longevity pathways of SIRT1 to foster endothelial vascular integrity during oxidant stress. Curr Neurovasc Res 8: 220-235, 2011

61. Koh PO: Nicotinamide attenuates the ischemic brain injury-induced decrease of Akt activation and Bad phosphorylation. Neurosci Lett 498: 105-109, 2011.

62. Yon JH, Daniel-Johnson J, Carter LB and Jevtovic-Todorovic V: Anesthesia induces neuronal cell death in the developing rat brain via the intrinsic and extrinsic apoptotic pathways. Neuroscience 135: 815-827, 2005.

63. Peltier J, O'Neill A and Schaffer DV: PI3K/Akt and CREB regulate adult neural hippocampal progenitor proliferation and differentiation. Dev Neurobiol 67: 1348-1361, 2007. 
64. Wyatt LA, Filbin MT and Keirstead HS: PTEN inhibition enhances neurite outgrowth in human embryonic stem cell-derived neuronal progenitor cells. J Comp Neurol 522: 2741-2755, 2014.

65. Ojeda L, Gao J, Hooten KG, Wang E, Thonhoff JR, Dunn TJ, Gao T and Wu P: Critical role of PI3K/Akt/GSK3 $\beta$ in motoneuron specification from human neural stem cells in response to FGF2 and EGF. PLoS One 6: e23414, 2011.

66. Luo HR, Hattori H, Hossain MA, Hester L, Huang Y, Lee-Kwon W, Donowitz M, Nagata E and Snyder SH: Akt as a mediator of cell death. Proc Natl Acad Sci USA 100: 11712-11717, 2003.

67. Song G, Ouyang G and Bao S: The activation of Akt/PKB signaling pathway and cell survival. J Cell Mol Med 9: 59-71, 2005.

68. Yeste-Velasco M, Folch J, Casadesús G, Smith MA, Pallàs M and Camins A: Neuroprotection by c-Jun NH2-terminal kinase inhibitor SP600125 against potassium deprivation-induced apoptosis involves the Akt pathway and inhibition of cell cycle reentry. Neuroscience 159: 1135-1147, 2009.

69. Wang W, Shi L, Xie Y, Ma C, Li W, Su X, Huang S, Chen R, Zhu Z, Mao Z, et al: SP600125, a new JNK inhibitor, protects dopaminergic neurons in the MPTP model of Parkinson's disease. Neurosci Res 48: 195-202, 2004.

70. Kuan CY and Burke RE: Targeting the JNK signaling pathway for stroke and Parkinson's diseases therapy. Curr Drug Targets CNS Neurol Disord 4: 63-67, 2005.
71. Walker CL, Walker MJ, Liu NK, Risberg EC, Gao X, Chen J and Xu XM: Systemic bisperoxovanadium activates Akt/mTOR, reduces autophagy and enhances recovery following cervical spinal cord injury. PLoS One 7: e30012, 2012.

72. Guan QH, Pei DS, Zhang QG, Hao ZB, Xu TL and Zhang GY: The neuroprotective action of SP600125, a new inhibitor of JNK, on transient brain ischemia/reperfusion-induced neuronal death in rat hippocampal CA1 via nuclear and non-nuclear pathways. Brain Res 1035: 51-59, 2005.

73. Han JY, Jeong EY, Kim YS, Roh GS, Kim HJ, Kang SS, Cho GJ and Choi WS: C-jun N-terminal kinase regulates the interaction between 14-3-3 and bad in ethanol-induced cell death. J Neurosci Res 86: 3221-3229, 2008.

74. Fan J, Xu G, Nagel DJ, Hua Z, Zhang N and Yin G: A model of ischemia and reperfusion increases JNK activity, inhibits the association of bad and 14-3-3 and induces apoptosis of rabbit spinal neurocytes. Neurosci Lett 473: 196-201, 2010.

75. Liao Z, Cao D, Han X, Liu C, Peng J, Zuo Z, Wang F and Li Y: Both JNK and P38 MAPK pathways participate in the protection by dexmedetomidine against isoflurane-induced neuroapoptosis in the hippocampus of neonatal rats. Brain Res Bull 107: 69-78, 2014.

76. Zheng S and Zuo Z: Isoflurane preconditioning induces neuroprotection against ischemia via activation of P38 mitogen-activated protein kinases. Mol Pharmacol 65: 1172-1180, 2004. 